\title{
Correction to: Basic Characteristics of Muse Cells
}

\author{
Shohei Wakao, Yoshihiro Kushida, and Mari Dezawa
}

\section{Correction to:}

\section{Chapter 2 in: M. Dezawa (ed.), Muse Cells,} Advances in Experimental Medicine and Biology 1103, https://doi.org/10.1007/978-4-431-56847-6_2

The below listed corrections have been carried out in the following pages of the current version:

1. In page 14, the legend of Fig. 2.1 "Trilineage differentiation ability of single Muse cell-derived cluster. The central phase-contrast microscopic image shows expanded cells emerging from the single Muse cell-derived cluster in gelatincoated culture dish. In expanded cells, cells positive for neurofilament, cytokeratin 7, smooth muscle actin, alpha-fetoprotein, and desmin were detected" is corrected to "SSEA-3(+) Muse cells differentiate into various kinds of ectodermal-, endodermal- and mesodermal-lineage cells in vitro and in vivo, either spontaneously or by cytokine induction."

2. In page 16 , section 2.2 , sub-section 2.2 .1 , the third paragraph, "Trilineage differentiation" was duplicated in the last sentence which is now corrected and the updated sentence now reads "Because single cell-derived pluripotent-like clusters are not formed, trilineage differentiation and self-renewal are"

3. In page 23, section 2.5, sub-section 2.5.1, the fourth paragraph, the word "uick" is incorrect. It is corrected to "quick".

4. In page 24, section 2.6, sub-section 2.6.1, Figure 2.8, the word "chorangiocytes" is incorrect. It is corrected to "Cholangiocytes".

5. In page 30 , section 2.8 , the ratio " $0.01 \sim 0.03 \%$ " is incorrect. It is corrected to "0.1 0.03\%". 